\title{
Genetic of dystonias according the new classification
}

\section{Genética das distonias de acordo com a nova classificação}

Carlos R. M. Rieder ${ }^{1,2}$

1 Universidade Federal do Rio Grande do Sul, Hospital de Clínicas de Porto Alegre, Unidade de Distúrbios do Movimento, Porto Alegre RS, Brazil;

2Universidade Federal Ciências da Saúde de Porto Alegre, Porto Alegre RS, Brazil.

Correspondence: Carlos R. M. Rieder; Rua Padre Chagas, 147 - conj. 602; 90570-080 Porto Alegre RS, Brasil; E-mail: carlosrieder@gmail.com

Conflict of interest:

There is no conflict of interest to declare.

Received 20 March 2015 Accepted 27 March 2015
$\mathrm{I}$ n 2013, a new classification of dystonia based in an international panel of experts was published in the journal of Movement Disorders ${ }^{1}$. This consensus provided an update on definition, phenomenology and classification of dystonia. By the new definition " $d y s$ tonia is a movement disorder characterized by sustained or intermittent muscle contractions, causing abnormal, often repetitive, movements, postures or both. Dystonic movements are typically patterned, twisting and may be tremulous". This new classification encompasses two axis, namely "clinical characteristics" (Axis 1) and "etiology" (Axis 2). The first axis focuses on clinical manifestations of dystonia and includes the four following characteristics: age at onset, body region affected, temporal aspects, and any associated clinical manifestations. The second axis focuses on etiology with two dimensions for inheritance and underlying neuropathology. To clinical purpose these two axis should never be viewed independently. Instead the goal of delineating the clinical phenomenology (Axis I) is to build a syndromic pattern that will help in dystonia etiology ${ }^{2}$.Regarding the age of onset earlier dystonia classification was based in two groups (early and late onset) with an age cutoff of 26 years. This was based in a study that divided at 26 years the age on discriminating patients with $\mathrm{DYT}^{3}$. This age discriminator was obviously not suitable for application to all dystonias. The new classification system has a spectrum of onset from pediatric to adult. That include at age onset: infancy (birth to 2 years); childhood (3-12 years); adolescence (13-20 years); early adulthood (21-40 years); late adulthood (40 years and older). The age at onset may give important clues to the underlying etiology $y^{2,4}$. Regarding the body distribution dystonia are described as focal, segmental, multifocal, generalized (with or without leg involvement) or hemidystonia. The body region affected has important value in guiding not only treatment decisions but also the etiological diagnosis. Temporal aspects which features had not been considered in the previous classification have been included. They includes manner of onset (acute or insidious), short-term variations in symptoms (diurnal, intermittent, or action induced), and longer-term variations in overall severity (static or progressive). Temporal aspects have important implications for diagnostic testing. For instance, diurnal patterns are suggestive of dopa-responsive dystonia, and intermittent patterns suggest one of the paroxysmal dyskinesia. Rapid-onset dystonia are less-common conditions but when present we should considered dystonia parkinsonism or glutaric aciduria ${ }^{2}$. Regarding associated clinical manifestation dystonia the new terminology uses "isolated dystonia" to describe cases in which dystonia is the only motor feature apart from tremor. The previously called dystonia plus syndromes (e.g. myoclonus-dystonia) are now called "combined dystonia", which refer to phenomenology rather than etiology. The Axis 2 that is based in presumed etiology are classified according the nervous system pathology (evidence of degeneration/structural lesions/neither or both) and heritability (inherited or acquired). Inherited dystonia can be sex linked or autosomal, dominant or recessive, or mitochondrial. When an etiology is not defined, it is classified as idiopathic (sporadic or familial). The commonly used primary and secondary terms are not used in the new classification. In fact, these terms are confused once they have been used to mean different things ${ }^{2,4}$. Primary dystonia has already been used either to refer to idiopathic dystonia or to nomine phenotypically isolated forms of dystonia, regardless of etiology. By these reasons, the term "primary" should be abandoned ${ }^{2}$. The term heredodegenerative that was used based on etiology was also excluded. This term included an enormous list of conditions that was not very practical 
to organize dystonia etiology. Furthermore, some disorders are hereditary, but are not degenerative. On the other hands, some disorders are degenerative but not hereditary ${ }^{2}$. New genetic technologies has markedly accelerated the process of genes discovery related to dystonias ${ }^{5}$. In this edition of Arquivos de Neuro-Psiquiatria, Camargo et al. ${ }^{6}$, present a review of genetics of dystonias based on the new classification. The authors present the inherit dystonias according to the clinical aspects of the new classification such as age at onset, if isolated or combined, and intermittent patterns. The main clinical aspects of childhood-onset and adolescent-onset isolated dystonias (DYT 1, 2, 6, 13, 17), adult-onset isolated dystonias (DYT 7, 21, 23, 24, 25), dystonias combined with parkinsonism (DYT 3, 5, 12, 16), dystonias combined with myoclonus and chorea (DYT 4, 11, 15) and combined paroxysmal dystonia (DYT 8, 10, 18, 19, 20) were reviewed by the authors. With the advances in genetic technologies such as exome and whole-genome, sequencing new genes underlying dystonia will be discovered ${ }^{5}$.Certainly idiopathic dystonia types will be reclassified in inherited forms once new genes will be recognized. However, phenotypic dystonia classification will be always important to recognize particular forms of dystonia and to conduct correct diagnostic and therapeutic strategies.

\section{References}

1. Albanese A, Bhatia K, Bressman SB, Delong MR, Fahn S,

Fung VS et al. Phenomenology and classification of dystonia: a consensus update. Mov Disord. 2013;28(7):863-73.

http://dx.doi.org/10.1002/mds.25475

2. Jinnah HA, Albanese A. The new classification system for the dystonias: why was it needed and how was it developed? Mov Disord Clin Pract (Hoboken). 2014;1(4):280-4. http://dx.doi.org/10.1002/mdc3.12100

3. Bressman SB, Sabatti C, Raymond D, Leon D, Klein C, Kramer PL et al. The DYT1 phenotype and guidelines for diagnostic testing. Neurology. 2000;54(9):1746-52.
Balint B, Bhatia KP. Dystonia: an update on

phenomenology, classification, pathogenesis and treatment. Curr Opin Neurol. 2014 Aug;27(4):468-76. http://dx.doi.org/10.1097/WC0.0000000000000114

5. Lohmann K, Klein C. Genetics of dystonia: What's known? What's new? What's next? Mov Disord. 2013 Jun 15;28(7):899-905. http://dx.doi.org/10.1002/mds.25536

6. Camargo $\mathrm{CH}$, Camargos ST, Cardoso FE, Teive HG. The genetics of the dystonias: a review based on the new classification of the dystonias. Arq Neuro-Psiquiatria. 2015;73(4):350-8. 